\title{
Combination of Tanacethum Partenium, 5-Hydrossitriptophan (5-Http) and Magnesium in the Prophylaxis of Episodic Migraine without Aura (AURASTOP ${ }^{\circledR}$ ) An Observational Study
}

\author{
Paola Zavarize $^{1}$, Gael Ngonga ${ }^{1}$, Alessandro Pezzini ${ }^{2}$, Giorgio Dalla Volta ${ }^{{ }^{*}}$ \\ ${ }^{1}$ Neurology Department, Istituto Clinico Città Di Brescia, Brescia, Italy \\ ${ }^{2}$ Department of Clinical and Experimental Sciences, Neurology Clinic, University of Brescia, Brescia, Italy
}

*Corresponding author: Giorgio Dalla Volta, Neurology Department, Istituto Clinico Città Di Brescia,Via Gualla, 25123 Brescia, Italy, E-mail: dalla@numerica.it

\begin{abstract}
Objective: The study aim is to verify whether treatment with a new combination of tanacethum partenium, 5-hydrossitriptophan (5-http) and magnesium (Aurastop ${ }^{\circledR}$ ) reduces headache frequency and intensity in patients suffering from episodic migraine without aura when used in migraine prevention. Methods: Forty patients, suffering from migraine without aura for at least 6 months with monthly frequency of 3 to 8 crises and presence of headache of 4 to12 days, were enrolled in this open study and treated orally with Aurastop twice daily for 3 months. The primary endpoint was reduction of migraine frequency (headache days per month) over an observation period of 3 months. The secondary endpoint was a composite of monthly frequency and intensity of pain crises, analgesics use (number of medications) and subjective change of pain intensity. Results: All the parameters significantly improved at the end of treatment with Aurastop. We observed a significant reduction of the number of headache days (from $8.8 \pm 2.0$ before treatment to $2.7 \pm 1.7$ post treatment, $p<0.001$ ), as well as of the number of attacks (from $5.0 \pm 1.2$ per month to $2.1 \pm 0.9$ per month, $\mathrm{p}<0.001$ ), of pain intensity (from Visual Analogic Scale [VAS] $6.9 \pm 1.0$ to $3.3 \pm 1.5, \mathrm{p}<$ 0.001 ), and of the number of analgesics assumed by each subject (from $8.5 \pm 1.6$ per month to $2.4 \pm 1.5$ per month, $\mathrm{p}<0.001$ ). No serious adverse events were observed. Conclusion: Though obtained in the setting of an open-trial, our findings suggest that the new combination of tanacethum partenium, 5-hydrossitriptophan (5-http) and magnesium (AURASTOP ${ }^{\circledR}$ ) is a promising approach for migraine prevention and warrant further investigation to confirm the safety and efficacy of this treatment.
\end{abstract}

Received Date: August 02, 2017

Accepted Date: August 10, 2017

Published Date: August 11, 2017

Citation: Dalla Volta, G., et al. Combination of Tanacethum Partenium, 5-Hydrossitriptophan (5-Http) and Magnesium in the Prophylaxis of Episodic Migraine without Aura (AURASTOP ${ }^{\circledR}$ )-An Observational Study. (2017) Int J Neuro Brain Dis 4(3): $1-4$.

DOI: $10.15436 / 2377-1348.17 .1630$

Keywords: Migraine without aura; NMDA receptors; Tanacetum parthenium

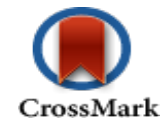

\section{Introduction}

A number of epidemiologic studies have consistently indicated that the prevalence of migraine is about $15-18 \%$ among women and $6 \%$ among men in Western Countries, with a pick between 22 ad 55 years ${ }^{[1]}$. Migraine is a chronic, disabling disease, with high impact in terms of social and economic costs ${ }^{[2]}$. Based on these characteristics, the WHO has recently included migraine among the 20 most disabling diseases affecting women between 15 and 45 years of age ${ }^{[3]}$. In particular, chronic headache has become the leading cause of disability and abuse of medical resources, accounting for up to $80 \%$ of the whole economic budget for public healthcare. In Italy, the direct and indirect costs of migraine have been estimated to be about 6 billion euros per year. The identification of migraine sufferers as well as the most appropriate management of these cases represents, therefore, a field of need. In this regard, it is generally accepted that a prophylactic treatment for migraine should be started for patients suffering disabling migraine for at least 4 days per month, or when pain crises do not respond to symptomatic medications.

The main goal of migraine prophylaxis is to reduce the frequency of attacks and patient disability, as well as to improve quality of life. A prophylactic therapy is considered effective when it reduces of at least $50 \%$ the frequency of migraine attacks. 
Agents that have been considered for migraine prophylaxis according to several national and international guidelines include, among the others, beta-blockers, calcium antagonists, serotonin reuptake inhibitors, anti-depressants, anti-epileptics, phytotherapics ${ }^{[4]}$, and magnesium.

Although the mechanisms of action of these molecules in migraine prevention are poorly known, their effects on the vessel wall, on neurons, as well as on specific neurotransmitters involved in migraine biology have been hypothesized based on numerous pre-clinical observations.

The biological origin of migraine, in fact, is likely multifactorial. Clinical studies suggest abnormal cortical, cerebrovascular, and immune functions. However, it is now well-accepted that the pain, persistence and throbbing features of migraine are mediated by increased sensitivity (i.e. sensitization) and the ensuing activation of sensory neurons innervating intracranial meninges and their related large blood vessels. These are the first-order neurons in the migraine pain pathway. Given the increasing importance placed on central and peripheral sensitization in the pathogenesis of migraine, the mechanisms of activation of trigeminal sensory afferents take on a central role. Sensitization results from the local release of neuropeptides that cause vasodilatation (mediated by calcitonin gene-related peptide) and an increase in vascular permeability (mediated by substance $\mathrm{P}$ and neurokinin $\mathrm{A}$ ) accompanied by mast cell activation. Mast cells, members of the innate immune system, participate in numerous physiological and pathophysiological conditions.

The emergence of a local inflammatory response in the meninges is widely viewed as a potential contributor to the activation and sensitization of meningeal nociceptors during migraine. Activation of resident immune cells such as mast cells, which are a prominent feature of the intracranial meninges, is likely to serve as a critical step in promoting enhanced excitability of meningeal nociceptors.

Activation and sensitization of primary afferent meningeal nociceptive neurons, the peripheral arm of the trigeminal-vascular system, appears to constitute one of the earliest events promoting the intracranial pain of migraine. These observations thus posit that meningeal mast cells, by virtue of their proximity both to meningeal blood vessels and nociceptive axons, may release a host of pro-inflammatory/algesic mediators responsible for the vasodilatatory phase of migraine associated with throbbing pain. These data suggest that controlling meningeal nociceptor excitability might be achieved by acting on mast cell activation.

A proposed mechanism of action involves parthenolide specifically binding to and inhibiting IкB kinase complex IKK $\beta$. IKK $\beta$ plays an important role in proinflammatory cytokine-mediated signaling. Parthenolide may also interact with TRPA1 nucleophilic sites, suggesting that feverfew antimigraine effect derives from its ability to target TRPA1. TRPA1 partial agonism, together with desensitization and nociceptor defunctionalization, ultimately resulting in inhibition of CGRP release within the trigeminovascular system, may contribute to the antimigraine effect of parthenolide ${ }^{[5-7]}$.

Glutamate is another neuropeptide likely involved in migraine pathogenesis. Actually, as it exerts an excitatory effect on first and second order neurons and it is involved in the activation of the trigeminovascular system, it is implicated in both migraine attack and the activity of the "migraine generator" in the brainstem. This makes it a key player in the activation and propagation of cortical spreading depression.

N-methyl-D-Aspartate (NMDA) is the post-synaptic glutamatergic receptor involved in central sensitization and cortical spreading depression, as demonstrated by its activation during migraine attacks ${ }^{[8]}$. NMDA receptors are activated by increased synaptic levels of glutamate, while they are inhibited by magnesium $^{[9-11]}$.

Glutamate levels are regulated by kynurenine ${ }^{[12]}$ which metabolizes 1-triptophan in kynurenic acid (KYNA) and quinolinic acid (QUINA). The NMDA receptor antagonist KYNA, in particular, inhibits glutamatergic pathway by blocking glutamate release and neurotransmission through its action on glycin Glu N1 binding site. It has been recently observed that in migraineurs the kynuretic pathway is shifted towards the conversion of KYNA in Antralinic Acid (ANA), as supported by the elevated plasma levels of ANA and the reduced concentration of KYNA and QUINA in these patients, with the consequent loss of inhibitory control on glutamate and its excitatory effects.

ANA has also a direct neurotoxic effect through the release of free radicals. Low plasma levels of KYNA may be considered a reliable marker of NMDA receptor activation.

Cerebral levels of KYNA can be increased by the assumption of its precursor $5-\mathrm{HTP}^{[13]}$.

Based on the mechanisms described above, the combination of 3 components, tanacetum parthenium, 5-HTP, and magnesium is expected to synergistically influence the biologic pathways involved in migraine pathogenesis, and, therefore, to have a therapeutic potential in migraine prevention.

\section{Patients and Methods}

Patients with episodic migraine as defined by the International Headache Society ${ }^{[14]}$ were consecutively recruited among those referring to the Headache Center of the Istituto Clinico Citta' di Brescia. Criteria for patients selection were: 1) age between 18 and 65 years; 2) diagnosis of migraine without aura, according to the International Headache Society; 3) migraine for at least 6 months with a monthly crisis frequency ranging from 3 to 8,4 ) headache duration ranging from 4 to 12 days per month, and 5) no other migraine preventive therapies. Patients with psychiatric co-morbidity, suffering from other types of acute or chronic pain, or with concomitant kidney failure, neurological or oncological diseases or pregnancy were considered non-eligible for the study.

The primary endpoint of the study was modification of migraine frequency (headache days per month) over an observation period of 3 months. The secondary endpoint was a composite of monthly frequency and intensity of pain crises, analgesics use (number of medications) and subjective change of pain intensity.

Patients who qualified for the study did not receive any prophylactic treatment for the first month since their enrollment in the study, then they received an association of Tanacetum Parthenium $150 \mathrm{mg}$ (1, $2 \mathrm{mg}$ of Partenolide), 5-HTP $20 \mathrm{mg}$, Magnesium $185 \mathrm{mg}$ (Dietary supplement Aurastop ${ }^{\circledR}$, Aesculapius Farmaceutici) twice daily for 3 months.

At baseline evaluation all patients underwent a thorough neurological examination and were carefully instructed on how to keep record of migraine attacks in their headache diary on 
a day-to-day basis. In particular, they were instructed to register: 1) attacks frequency, pain intensity (using the VAS scale, ranging from 1 to 10) and duration; 2) response to analgesics based on a self-rate scale ranging between 1 (minimum response) and 5 (maximum response) over the time period (1 month) with no preventive therapy and that ( 3 months) taking Aurastop. Safety was evaluated by treatment discontinuation rate and the occurrence of serious and otherwise adverse events.

\section{Statistical Analysis}

Categorical variables are reported as counts and percentages. Dependent variables were compared by McNemar's $\chi 2$ analysis. Wilcoxon's signed rank test was used to compare migraine characteristics before and after treatment. Statistical analyses were performed using SPSS 21.0 (IBM SPSS Statistics 2013, Armonk, NY, USA).

\section{Results}

Overall, 40 patients (15 males/and 25 females; mean age, 35, 6 years, range, 19 - 54) were enrolled in the study. One out of the 40 patients withdrew after 1 month of treatment because of non-improvement and one was lost to follow-up.

The results of the analysis are summarized in Table 1 . We observed a significant reduction of the number of headache days (from $8.8 \pm 2.0$ before treatment to $2.7 \pm 1.7$ post treatment, $\mathrm{p}<0.001$ ), as well as of the number of attacks (from $5.0 \pm 1.2$ per month to $2.1 \pm 0.9$ per month, $\mathrm{p}<0.001$ ), of pain intensity (from VAS $6.9 \pm 1.0$ to $3.3 \pm 1.5, \mathrm{p}<0.001$ ), and of the number of analgesics assumed by each subject (from $8.5 \pm 1.6$ per month to $2.4 \pm 1.5$ per month, $\mathrm{p}<0.001$; Figure 1 ). None of the patients reported any treatment-related adverse events.

Table 1: Migraine characteristics before and after prophylactic treatment with Aurastop.

\begin{tabular}{|l|l|l|l|}
\hline Migraine characteristics & Baseline & $\begin{array}{l}\text { Post- } \\
\text { treatment }\end{array}$ & p-value \\
\hline Pain intensity (0 to 10) & $6.9 \pm 1.0$ & $3.3 \pm 1.5$ & $<0.001$ \\
\hline Number if attacks/month & $5.0 \pm 1.2$ & $2.1 \pm 0.9$ & $<0.001$ \\
\hline Number of days/month & $8.8 \pm 2.0$ & $2.7 \pm 1.7$ & $<0.001$ \\
\hline Number of analgesics & $8.5 \pm 1.6$ & $2.4 \pm 1.5$ & $<0.001$ \\
\hline Analgesics (yes/no) & $41(100.0)$ & $35(89.7)$ & 0.125 \\
\hline
\end{tabular}

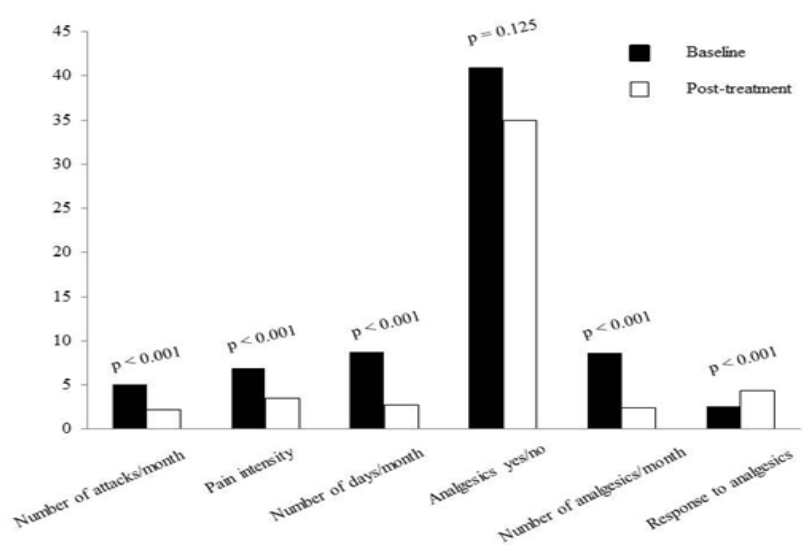

Figure 1: Comparison of individual migraine characteristics according to treatment-phase.
Another notable finding derived from the analysis of the headache diaries, was that the results at the 3-month time point reported above were already detectable after one month of prophylactic therapy with Aurastop.

\section{Discussion}

The results of the present study highlight the efficacy and the safe profile of the combination of Tanacetum Parthenium, 5-HTP and magnesium in migraine prophylaxis. Both, the frequency and the duration of migraine attacks as well as the intensity of pain improved significantly in most of the patients treated. These findings, in addition to the more favorable response to analgesics and the need of a reduced number of these medications in patients receiving Aurastop lead to the speculation that the 3 molecules in combination might act synergistically on different pathways involved in migraine biology: neurogenic inflammation, neural transmission, and central sensitization. The well-known phenomenon of Cortical Spreading Depression (CSD), involved in the pathogenesis of both migraine without aura and migraine with aura, has been consistently related to the status of inter-critical cortical brain hyperexcitability of migraineurs. This emphasizes the physiologic role of magnesium in the regulation of such a neuronal excitability. Among the many actions, intracellular magnesium acts as a physiologic calcium-antagonist, thus reducing the toxic effects of calcium, whereas suboptimal concentrations of magnesium favor free radical accumulation within the cell, which, in turn, may trigger a migraine attack. Furthermore, magnesium inhibits NMDA receptors, which are involved in the glutamate-dependent excitatory pathways at the basis of the neurogenic inflammation. Moreover, brain concentration of kynurenic acid (Kyna), a tryptophan derivative which further acts as an endogenous NMDA receptor antagonist, increases with increasing serum levels of its precursor 5HTP. Giving 5HTP as a drug may, therefore, increase Kyna levels and influence Kyna pathways, leading to the inhibition of peripheral NMDA receptors and the consequent activation of the trigeminovascular system, as well as of CSD.

Finally, TRPA1 and NMDA receptors with glutamate and calcitonin-gene-related peptide (CGRP) play a key role in neurogenic inflammation, which leads to the sensitization of trigeminal nucleus caudalis in the lower brainstem and upper cervical cord and, consequently, of all structures involved in the central transmission of nociceptive information.

Molecules proved to be migraine generators act as TRP receptors activators. These, in turn, generate neurogenic inflammation leading to painful attacks through the release of CGRP from perivascular nerve terminals. Such a cascade might be interrupted by partenolide, a TRP receptors inhibitor. Partenolide is, moreover, a powerful inhibitor of nitric oxide synthase and, consequently, of nitric oxide (NO) production. All the findings observed in the present study can, therefore, be interpreted as clinical manifestation of the interference with the phenomenon of central sensitization, as well as with the central transmission of nociceptive information at synaptic level, TRPA1 channels, and NMDA receptors determined by Aurastop. 


\section{Conclusion}

In spite of the methodological limitations inherent in the present pilot, observational study lacking a control group, our findings emphasize the potential influence of the combination of different molecules on the complex biologic mechanisms causing migraine. In particular, tanacetum parthenium +5 -HTP + Magnesium appear as a promising association of molecules for migraine prophylaxis with an excellent safety profile. Further clinical trials are warranted in order to investigate the potentials of this compound in preventing migraine, either given alone or in combination with other molecules.

\section{References}

1. Lipton, R.B., Bigal, M.E., Diamond, M., et al. Migraine Prevalence, Disease Burden, and the Need for Preventive Therapy. (2007) Neurol 68(5): 343-349.

Pubmed I Crossref I Others

2. Bloudek, L.M., Stokes, M., Buse, D.C., et al. Cost of Healthcare for Patients with Migraine in Five European Countries: Results from the International Burden of Migraine Study (IBMS). (2012) J Headache Pain 13(5): 361-378.

Pubmed I Crossref I Others

3. Steiner, T.J., Birbeck, G.L., Jensen, R., et al. The Global Campaign, World Health Organization and Lifting the Burden: Collaboration in Action. (2011) J Headache Pain 12(3): 273-274.

Pubmed I Crossref I Others

4. Rajapakse, T., Pringsheim, T. Nutraceuticals in Migraine: A Summary of Existing Guidelines for Use. (2016) Headache 56(4): 808-816. Pubmed I Crossref I Others

5. Diener, H.C., Pfaffenrath, V., Schnitker, J., et al. Efficacy and Safety of $6.25 \mathrm{mg}$ t.i.d. Feverfew CO2-Extract (MIG-99) in Migraine Prevention-A Randomized, Double-Blind, Multicentre, Placebo-Con- trolled Study. (2005) Cephalalgia 25(11): 1031-1041.

Pubmed I Crossref I Others

6. Tassorelli, C., Greco, R., Morazzoni, P., et al. Parthenolide Is the Component of Tanacetum parthenium That Inhibits Nitroglycerin-Induced Fos Activation: Studies in an Animal Model of Migraine. (2005) Cephalalgia 25(8): 612-621.

Pubmed I Crossref I Others

7. Materazzi, S., Benemei, S., Fusi, C., et al. Parthenolide Inhibits Nociception and Neurogenic Vasodilatation in the Trigeminovascular System by Targeting the TRPA1 Channel. (2013) Pain 154(12): 2750-2758. Pubmed I Crossref I Others

8. Begon, S., Pickering, G., Eschalier, A., et al. Role of Spinal NMDA Receptors, Protein Kinase C and Nitric Oxide Synthase in the Hyperalgesia Induced by Magnesium Deficiency in Rats. (2001) Br J Pharmacol 134(6): 1227-1236.

Pubmed I Crossref I Others

9. Sun-Edelstein, C., Mauskop, A. Role of Magnesium in the Pathogenesis and Treatment of Migraine. (2009) Expert Rev Neurother 9(3): 369-379.

Pubmed I Crossref I Others

[10] Mody, I., Lambert, J.D. and Heinemann, U. Low Extracellular Magnesium Induces Epileptiform Activity and Spreading Depression in Rat Hippocampal Slices. (1987) J Neurophysiol 57: 869-888.

Pubmed I Crossref I Others

[11] Teigen, L., Boes, C.J. An Evidence-Based Review of Oral Magnesium Supplementation in the Preventive Treatment of Migraine. (2015) Cephalalgia 35(10): 912-922.

Pubmed I Crossref I Others

12. Chauvel, V., Vamos, E., Pardutz, A., et al. Effect of Systemic Kynurenine on Cortical Spreading Depression and Its Modulation by Sex Hormones in Rat. (2012) Exp Neurol 236(2): 207-214.

Pubmed I Crossref I Others

13. Curto, M., Lionetto, L., Negro, A., et al. Altered Kynurenine Pathway Metabolites in Serum of Chronic Migraine Patients. (2015) J Headache Pain 17: 47.

Pubmed I Crossref I Others

14. The International Classification of Headache Disorders. $3^{\text {rd }}$ Edition (Beta Version). (2013) Cephalalgia 33(9): 629-808.

Pubmed I Crossref I Others
Online ISSN: $2377-1348$

Journal Title: International Journal Neurology and Brain Disorders Journal Short Name: Int J Neurol Brain Disord
Ommega Online Publishers

E-mail: neurology@ommegaonline.com

Website: www.ommegaonline.org 